\title{
Simultaneous knockdown of APRIL via multiple shRNAs reduces the malignancy of SW480 cells
}

\author{
HAIQUAN LI ${ }^{1}$, KEKE SHAO ${ }^{2}$, JINGCHUN WANG ${ }^{1}$, GUIHUA WANG $^{1}$, \\ $\mathrm{JIN} \mathrm{XU}^{1}$, JINDE CAO ${ }^{1}$, SHAOQING JU ${ }^{1,3}$ and HUIMIN WANG ${ }^{1}$ \\ ${ }^{1}$ Department of Clinical Laboratory Center, Affiliated Hospital of Nantong University, Nantong Medical College, \\ Nantong University, Nantong 226001; ${ }^{2}$ Clinical Laboratory, Yancheng City No. 1 People's Hospital, Yancheng 224001; \\ ${ }^{3}$ Surgical Comprehensive Laboratory, Affiliated Hospital of Nantong University, Nantong 226001, P.R. China
}

Received March 26, 2012; Accepted June 8, 2012

DOI: $10.3892 /$ or.2012.1980

\begin{abstract}
A proliferation-inducing ligand (APRIL) is a key factor involved in the tumor development and progression in some tumor tissues and cells. Its overexpression and as gene target in SW480 colon carcinoma cells was confirmed in our previous study. To seek a more potent way to treat colon carcinoma using a gene therapy method, herein, we constructed a multiple short hairpin RNA (shRNA) expression vector containing four shRNAs against the APRIL gene in SW480 cells. APRIL expression levels and cell biological behavior were detected after transfection with different kinds of vectors. As expected, we found that our multiple shRNA vector produced a more significant knockdown effect of APRIL than the vectors containing only one APRIL shRNA. Furthermore, our findings indicate that silencing APRIL expression in SW480 cells decreased their malignancy by reducing proliferation, invasion and adhesion, as well as inducing apoptosis. Based on our findings, vectors containing multiple shRNAs to silence the expression of APRIL may be exploited as a novel therapeutic strategy for tumors.
\end{abstract}

\section{Introduction}

APRIL (a proliferation-inducing ligand), also known as TNFSF13, TALL-2 and CD256, a member of tumor necrosis factor (TNF) superfamily, was first discovered and successfully cloned by Hahne et al in 1998 (1,2). It can induce cell proliferation and overexpressed in most tumor tissues or cells, especially in the digestive system carcinomas, such as colon carcinoma, pancreatic cancer, gastric cancer, hepatoma and esophageal carcinoma (3-8). APRIL plays an essential role in the occurrence and development of some tumors, including colon carcinoma. Given its great participation in these tumors, some researchers

Correspondence to: Dr Huimin Wang, Department of Clinical Laboratory Center, Affiliated Hospital of Nantong University, Nantong Medical College, Nantong University, Nantong 226001, P.R. China E-mail: ntfyjyk@pub.nt.jsinfo.net

Key words: RNA interference, multiple shRNAs, APRIL, SW480, colon carcinoma have reported that APRIL gene can be exploited as a therapeutic target (9).

RNA interference (RNAi), a cellular intrinsic gene silencing mechanism, is a generally identified biological process in which specific sequence of short dsRNA is knocked down in the genes complimentary to the dsRNA (10). It was initially found in lower organisms such as plants and worms, and later it was recognized that short dsRNA can specifically decrease the levels of the mRNA through the RNAi pathway in mammalian systems as well (11). Moreover, RNAi has been developed to knock down gene expression in a variety of mammalian cells. Widespread application of this technique has been reported in target validation studies for drug research, while its potential use for therapeutic purposes is still under debate due to its limited efficiency (12). In order to maximize the efficiency of the vector-based siRNA approach, Wang et al have developed vectors containing multiple ( $\leq 6)$ tandem siRNA expression cassettes, which can achieve most effective knockdown of genes (13).

SW480 is screened from several colon carcinoma cell lines, and its overexpression of APRIL was confirmed in our previous study. Ding et al, recently indicated that APRIL functions in the tumor development of biological behavior of colon carcinoma cells (14). In this study, we constructed a single vector containing four shRNAs that target four regions of the APRIL gene expressed in SW480 cells. Four shRNA vectors each containing one shRNA were constructed as a control. The APRIL protein and APRIL mRNA were observed after transfection with our different shRNA vectors. As a result, we found that using a single vector containing four shRNAs produced a more effective inhibition of APRIL than the vectors containing only one shRNA. Furthermore, biological behaviour of the studied cells transfected with multiple shRNAs are much more depressed than the cells containing a single one, and the malignancy of the carcinoma cells was reduced. It suggests that vectors containing multiple shRNA targeting APRIL may serve as a more useful therapeutic target in malignant colon carcinomas.

\section{Materials and methods}

ShRNA preparation and plasmids construction. Four target regions of APRIL gene were selected (GenBank accession no. NM_003808.3) using the online tool www.ambion.com. Then 
we designed four pairs of DNA oligonucleotides for the following steps, respectively named as sh644, sh1451, sh1938, and sh2231 (Fig. 1C) according to the site of the target region of APRIL gene. Four pairs of chemically-synthesized shRNA sequences were respectively inserted into linearized pGenesil1-T vector (Fig. 1A) between BamHI and HindIII in the multiple cloning sites (MCS), yielding pGenesil1-T1 (pGsh644), pGenesil1-T2 (pGsh1451), pGenesil1-T3 (pGsh1938), pGenesil1-T4 (pGsh2231). These four pairs of oligonucleotide sequences were constructed into pGenesil4-T (pG4) vector (Genesil Corp., China) (Fig. 1B) which has four different promoters (mU6, hU6, h7SK, and hH1) as the methods stated by Gou et al (27). We use the HK vector in which was inserted a sequence (5'-GAVTTCATAAGGCGCATGC-3') with limited homology to any known cDNA sequence in the human and mouse genomes as a negative control. We used both restriction enzyme digestion and DNA sequencing to make sure all the inserted sequences are correct.

Cell culture and plasmid transfection. SW480, the colon carcinoma cell line, was purchased from Academy of Life Science, Shanghai, China. It was cultured in RPMI-1640 medium (Gibco) supplemented with 10\% fetal bovine serum (Sijiqing, Hangzhou), of L-glutamine and antibiotics (streptomycin and penicillin) at $37^{\circ} \mathrm{C}$ in humidified incubator containing $5 \% \mathrm{CO}_{2}$. SW480 cells were seeded at a concentration of $2 \times 10^{6}$ per well (6-well, Coring) and the next morning they were transiently transfected with pGsh644, pGsh1451, pGsh1938, pGsh2231, pG4 and HK vectors with Lipofectamine 2000 (Invitrogen, Jefferson City, MO, USA) according to the manufacturer's instructions. After $48 \mathrm{~h}$ of transfection, cells were harvested for the following analysis.

Analysis of APRIL mRNA level by RTFQ-PCR. Forty-eight hours after transfection, mRNA was extracted from total $2 \times 10^{6}$ cell and reversely transcribed into cDNA by using RevertAid (Fermentas) accoring to the manufacturer's instructions. Then, real-time fluorescence quantitative PCR (RTFQ-PCR) was performed by using Applied Biosystems 7500 in the volume of the $30 \mu \mathrm{l}$ reaction system (PrimeScrip, Takara), containing $10 \mu \mathrm{M}$ of primers for APRIL amplification. The primer sequences were as follows: 5'-ACTCTCAGTTGCCCTCTGGTTG-3' (sense) and 5'-GGAACTCTGCTCCGGGAGACTC-3' (antisense). We also used GAPDH amplification as a control for normalization, and the primers used were: 5'-ACGCATTTGGTCGTATTGGG-3' (sense) and 5'-TGATTTTGGAGGGATCTCGC-3' (antisense). The PCR protocol was: initial denaturation for 2 min at $93^{\circ} \mathrm{C}$, 40 cycles at $93^{\circ} \mathrm{C}$ for $5 \mathrm{sec}, 62^{\circ} \mathrm{C}$ for $35 \mathrm{sec}$ and $86^{\circ} \mathrm{C}$ for $1 \mathrm{sec}$. Specificity of amplification product was checked by dissociation curves. Each sample was detected in triplicate. Results were analyzed with Applied Biosystems 7500 software, version 2.0.1.

Western blot analysis. After 48-h transfection, cells were washed three times with phosphate-buffered salt solution (PBS), lysed in $50 \mu 1$ of ice-cold protease inhibitor cocktail and RIPA buffer (Beyotime, Nantong, China), and then lysates were centrifugated at $14,000 \times \mathrm{g}$ for $10 \mathrm{~min}$ at $4^{\circ} \mathrm{C}$ to remove the cell debris and the concentration of protein was determined by Nano photometer. Protein lysates were separated by $10 \%$ sodium dodecylsulfate-polyacrylamide gel electrophoresis (SDS-PAGE) and then transferred onto nitrocellulose membranes. The membranes were blocked with Tris-buffered saline containing $0.1 \%$ Triton X-100 (TBST) non-fat milk overnight at $4^{\circ} \mathrm{C}$, afterwards incubated with anti-human APRIL antibody and $\beta$-actin (Santa Cruz) at $4^{\circ} \mathrm{C}$ overnight. After washing with TBST, the membrane was incubated with HRP-conjugated mouse immunoglobulin at room temperature for $2 \mathrm{~h}$. The signal was detected using the enhanced chemiluminescence method (ECL). $\beta$-actin was used as the internal control. Protein was evaluated by using Bandscan Image software for gray scale scan.

CCK-8 cell growth viability assay. Cells at a concentration of $5 \times 10^{3}$ per well were seeded in the 96 -well plate and incubated at $37^{\circ} \mathrm{C}$ in a humidified atmosphere containing $5 \% \mathrm{CO}_{2}$ for $24 \mathrm{~h}$. Twelve hours after transfection, we added $10 \mu \mathrm{l}$ of the Cell Counting kit-8 (CCK-8) solution to each well of the plate. Two hours later, absorbance was recorded at $450 \mathrm{~nm}$ using Thermo Multiskan MK3. Likewise, we subsequently recorded the absorbance 8, 24 and $36 \mathrm{~h}$ later after successful transfection. The cell growth viability curve was recorded.

In vitro adhesion and invasion assay. SW480 cells $\left(10^{6}\right.$ cells $\left./ \mathrm{ml}\right)$ transfected with the vectors indicated above were incubated in the wells coated with human collagen type IV (Chemicon, USA) at $37^{\circ} \mathrm{C}$ for $45 \mathrm{~min}$ in a $\mathrm{CO}_{2}$ incubator. Thereafter, $100 \mu \mathrm{l}$ of $0.2 \%$ crystal violet was added to each well and incubated for $5 \mathrm{~min}$ at room temperature. The absorbance at $540 \mathrm{~nm}$ was detected in the stained cells with $100 \mu \mathrm{l}$ of solubilization buffer (50 $\mu \mathrm{l} 0.1 \mathrm{M}$ $\mathrm{NaH}_{2} \mathrm{PO}_{4}, \mathrm{pH} 4.5$ and $50 \mu 150 \%$ ethanol) to evaluate the capability of cell adhesion. The cell invasion assay was performed in an invasion chamber (Corning, Transwell), a 24-well tissue culture plate with 12-well culture inserts. Cell suspensions transfected with different vectors were added to the interior of the inserts with $300 \mu \mathrm{l}$ serum-free medium, $500 \mu \mathrm{l}$ of medium containing $10 \%$ fetal bovine serum to the lower chamber and incubated in a tissue culture incubator at $37^{\circ} \mathrm{C}$ in $5 \% \mathrm{CO}_{2}$ for $72 \mathrm{~h}$ to allow the cells to migrate. Cells on the upper surface of the filter were removed by wiping with a cotton swab. Migrated cells on the lower side of the filters were fixed and stained with hematoxylin and eosin. The number of migrated cells was counted under a light microscope.

Flow cytometry analysis. The SW480 cells were transfected with shRNA expression vectors in 6-well plates after washing with phosphate-buffered saline (PBS) three times. After 48-h transfection, cells were harvested and washed twice in cold PBS, discarding the supernatant and resuspended at $1 \times 10^{6}$ cells $/ \mathrm{ml}$ in binding buffer and placed in a $100 \mu \mathrm{l}$ tube. Thereafter $5 \mu \mathrm{l}$ annexin-V and $10 \mu \mathrm{l}$ propidium iodide (PI) were added to each tube, and the stained cells were analyzed by flow cytometry (FCM) using BD FACSDiva software (BD).

\section{Results}

Multiple shRNAs simultaneously inhibit APRIL gene expression. Four single shRNA vectors containing only one APRIL shRNA (pGsh644, pGsh1451, pGsh1938 and pGsh2231) and one multiple shRNAs vector having all four shRNAs (pG4) were successfully constructed which was confirmed by both 
A

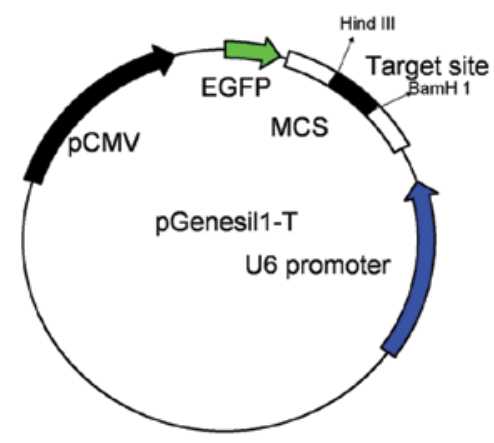

B

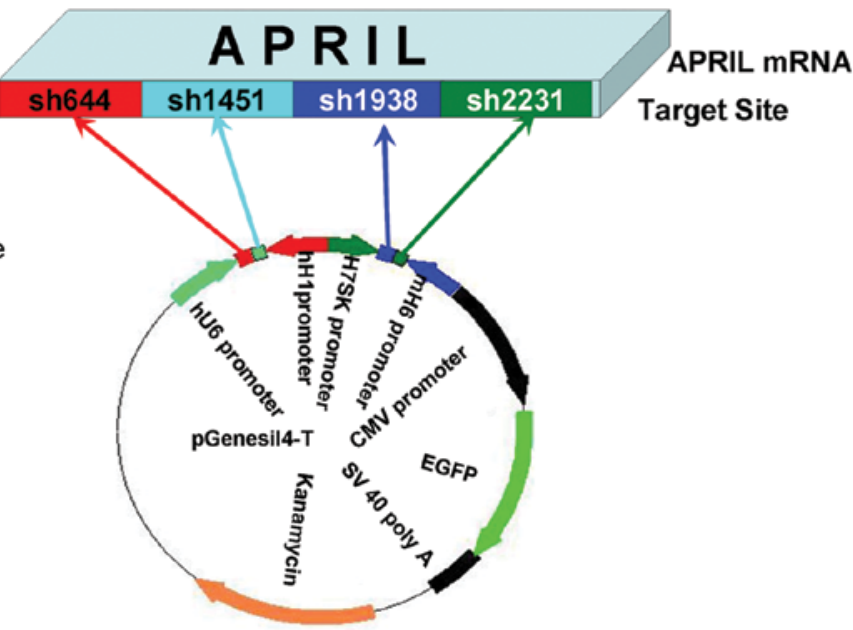

C

\begin{tabular}{|l|c|}
\hline shRNA & sequence \\
\hline sh644 & 5'-CAACCUUCUUCCCUUCUGC UUCAAGACG GCAGAAGGGAAGAAGGUUG UU-3' \\
\hline sh1451 & 5'-ACUUAACCUCUCUCCACAU UUCAAGACG AUGUGGAGAGAGGUUAAGU UU-3' \\
\hline sh1938 & 5'-GAAGCCUUAUCCUACGUCC UUCAAGACG GGACGUAGGAUAAGGCUUC UU-3' \\
\hline sh2231 & 5'-GCCCUGCUUAAAGUUAAAU UUCAAGACG AUUUAACUUUAAGCAGGGC UU-3' \\
\hline
\end{tabular}

Figure 1. The shRNA targeting sites and the shRNA expression vector structure and sequences. (A) The target site of APRIL mRNA for shRNA and the structure of expression vector construct of pGenesil4-T. (B) The circular structure of expression vector construct of pGenesil1-T and four representative hairpin structures of shRNA. (C) Four pairs of representative short hair-pin RNA sequences were chemically synthesized and are shown in the table.

restriction enzyme digestion and DNA sequencing. We used coexpression of EGFP (enhanced green fluorescence protein) which had been cloned into the vector to detect the transfection efficiency of the APRIL shRNA vectors. After 24-h transfection, $>75 \%$ of cells were EGFP-positive after transfection with all vectors (Fig. $2 \mathrm{~A}$ ).

To evaluate the inhibition efficiencies of APRIL mRNA expression, RTFQ-PCR was performed after transfection, the APRIL mRNA expression in SW480 cells transfected with APRIL multiple shRNA was reduced by nearly $80 \%$. While the single shRNA vectors decreased APRIL mRNA levels by 55.9, 46.5, 49.5 and 44\%, respectively (Fig. 2B), as compared with untransfected SW480 cells and negative shRNA control transfected ones $(\mathrm{P}<0.05)$.

Then we studied the effects of the pGshRNA vectors on APRIL protein expression in SW480 cells by western blot analysis. The APRIL protein expression demonstrated a significant reduction in the multiple shRNAs expression vector (pG4), decreasing APRIL protein level by $85.7 \%$ compared to untransfected ones $(\mathrm{P}<0.05)$. The single vectors (pGsh644, pGsh1451, pGsh1938 and pGsh2231) reduced APRIL protein levels by $49-57 \%$ (Fig. 2C). In contrast, no obvious changes were observed between the negative control group and the untransfected group.

APRIL knockdown strongly depresses SW480 cell proliferation, adhesion and invasion through multiple shRNAs. Cell proliferation, invasion, adhesion and apoptosis are essential cellular events that contribute to tumor malignancy. We compared the

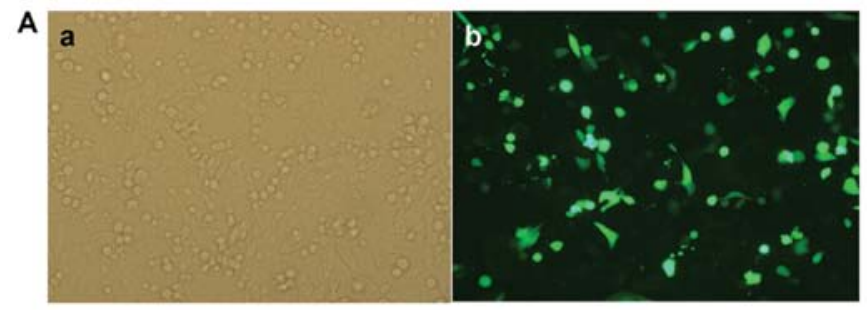

B

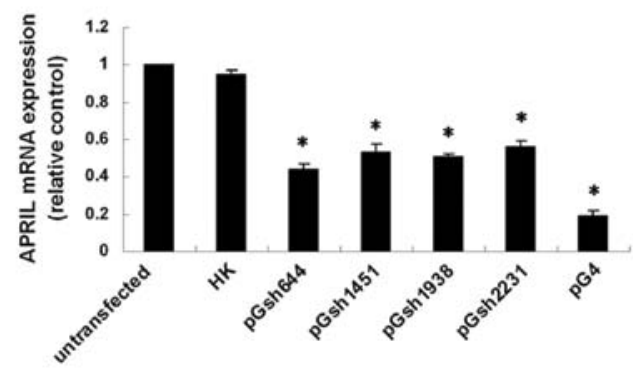

C

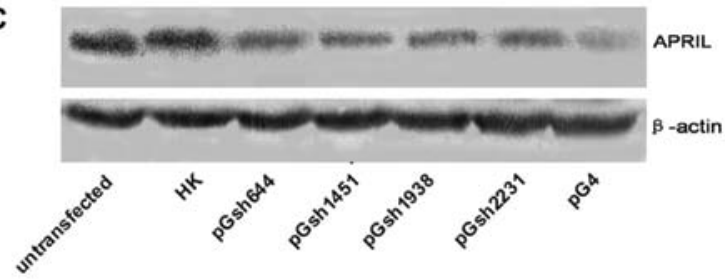

Figure 2. Expression of APRIL mRNA and protein after being transfected with shRNA. (A) After 24-h transfection, light (a) and fluorescent microphotos (b) were taken. (B) APRIL mRNA expression was detected by real-time PCR " $\mathrm{P}<0.05$, compared with untransfected group. Representitive data from three independent experiment are shown. (C) Western blotting was used to analyze the APRIL protein expression of SW480 cells transfected with shRNA. 

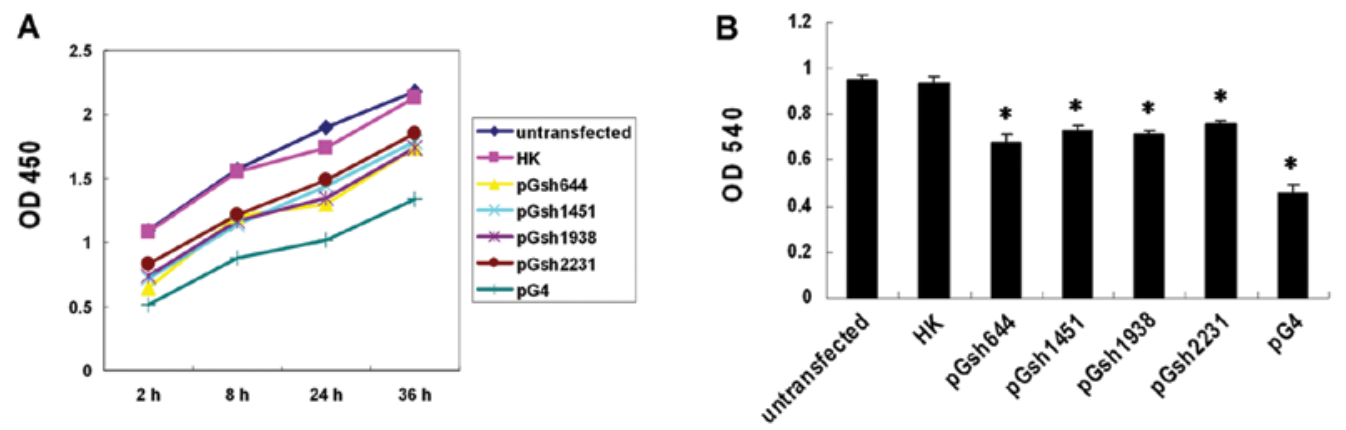

C

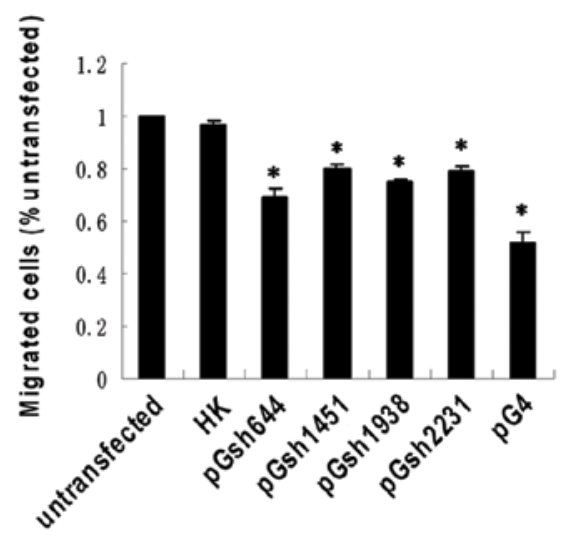

D

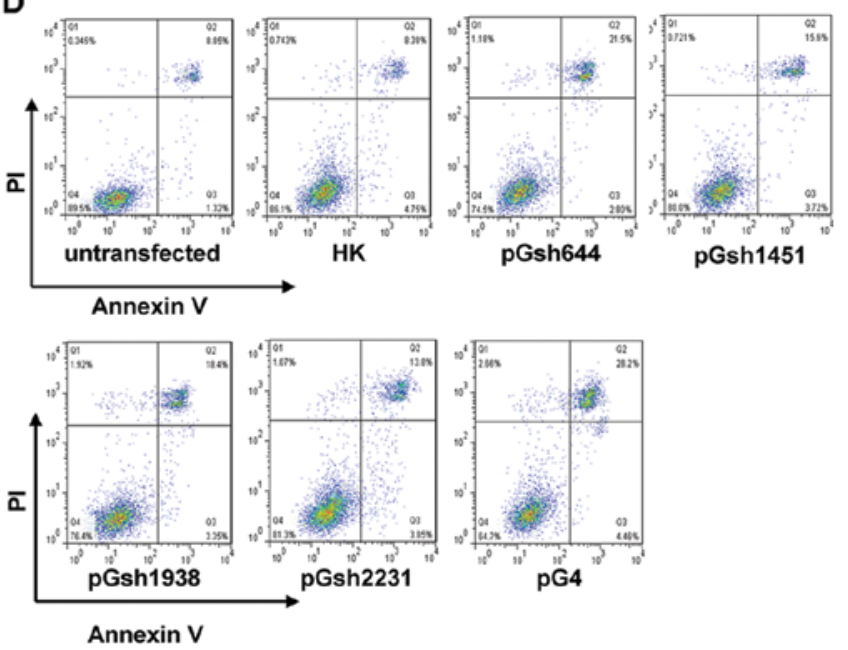

Figure 3. APRIL knockdown suppresses the growth of SW480 cells. (A) Proliferation of SW480 cells transfected with shRNA expression vectors were analyzed by CCK-8 kit. Absorbance at $450 \mathrm{~nm}$ was measured at different time-point using a microplate reader. Each group contained three cell types. (B) Adhesion of SW480 cells transfected with shRNA to dishes coated with fibronectin were evaluated. Each group contained three cell types. (C) Matrigel invasion was evaluated by using transwell invasion chambers. Number of cells migrated was counted in five fields for each experimental group and averanged. (D) SW480 cells were treated with shRNA expression vectors for $48 \mathrm{~h}$, harvested and analyzed by flow cytometry (FCM). These experiments were repeated three times ("P $<0.05$, compared with untransfected vector).

level of perliferation in SW480 cells transfected with different shRNA vectors using the CCK- 8 assay at 4 different time-points $12 \mathrm{~h}$ after transfection. We found the number of viable cells transfected with pG4 were significantly decreased compared with the single one at all different time-points (Fig. 3A). The SW480 cell adhesion ability was investigated with the absorbance at 540 $\mathrm{nm}$. The multiple vector transfected cells significantly reduced the adhesion rate by $44.6 \%$, compared to the untransfected cells (Fig. 3B). In our invasion assay, cells transfected with multiple shRNAs invaded at $<52 \%$ of the untransfected group (Fig. 3C). In all these studies, no significant differences in proliferation, adhesion and invasion were observed between cells transfected with HK and untransfected cells. These data indicate that multiple shRNAs are much more potent in inhibition of cellular biological behavior than the single one.

Silencing APRIL expression by multiple shRNAs induces colon carcinoma cell apoptosis significantly. We examined apoptosis using flow cytometry (FCM) and found that apoptosis was increased in cells transfected with single pGshRNA as compared to the untreated cells, while the multiple shRNA (pG4) vector induced the highest level of apoptosis (32.6\%) in SW480 cell line (Fig. 3D). Taken together, the results demonstrate that the multiple shRNA vector targeted APRIL had a more significant knockdown effect on colon carcinoma cells than the single ones.
Furthermore, our data indicate that APRIL may be a useful therapeutic target in malignant colon carcinoma.

\section{Discussion}

APRIL, as a member of the TNF superfamily, has the classical TNF fold, which can recognize and bind to cysteine-rich domains (CRD) of the TNF receptor (TNFR) family (15). It has been proven that APRIL has an substantial proliferationinducing effect, including tumor cell growth. Previous studies reported that APRIL has two definite receptors, BCMA and TACI, and more importantly, HSPG, which served as a third receptor or co-receptor of APRIL, is a predominant receptor for APRIL in the SW480 colon carcinoma cell line (2,16-18). Studies comfirmed that HSPG plays an important role in a wide variety of biological responses and processes such as adhesion, migration, proliferation, embryonal development, differentiation, morphogenesis, angiogenesis and blood coagulation (19-22). In several colon carcinoma cell lines, including the SW480 cell line, APRIL and HSPG gene overexpression have been found. Ding et al suggested that low levels of APRIL play an essential role in cellular senescence via an HSPG-dependent signaling pathway in SW480 (23). Thus, a possible interpretation is that the metastasis-promoting ability of APRIL is associated with the engagement of HSPG. But in 
this study, we put the APRIL gene in the first place to investigate the efficiency of multiple shRNAs. Studies have found that APRIL could induce not only proliferation but metastases of the tumor cells (14) implying that downregulation of APRIL expression by transfection of shRNA could reverse cell proliferation and other biological behavior. These results have been confirmed in our studies.

RNA interference (RNAi) is a sequence-specific posttranscriptional gene silencing mechanism, which has become a powerful tool for investigating gene function by reverse genetics $(10,24)$. Recently, this new approach has been widely used in the study of gene therapy for cancer by vector-based strategies for delivery of siRNA into mammalian cells $(25,26)$. Howerer, efficient knockdown of some mRNA has been difficult to achieve by the vector that contain only one siRNA, herein, several of the shRNAs have been used to target multiple sites within a single mRNA to maximize knockdown efficiency (27). Based on this point, we constructed a short hairpin RNA (shRNA) expression vector containing four shRNAs to knockdown the APRIL gene in SW480 cells to observe the tumor development of colorectal cancer. As expected, we found that the vector expressing four shRNAs against APRIL gene was more potent at silencing APRIL gene expression than the individual single shRNA expression plasmids. This finding is consistent with reports on other genes (28), indicating a new concept for gene therapy using a combination of four RNAi sites that are targeted towards a specified gene.

Our results demonstrated that multiple shRNA expression vector indeed significantly inhibited APRIL gene expression. Furthermore, silencing APRIL expression using multiple shRNAs in colon carcinoma cells substantially depressed malignancy of the tumor cells by reducing proliferation, adhesion and migration, and enhancing apoptosis. This finding suggests a novel way to perform gene therapy for tumors.

Colon carcinoma is one of the most prevalent and fatal cancers in the western world (29), and in China, the rate of colon carcinoma is increasing. Despite the advances in surgery, radiotherapy and chemotherapy, the long-term survival rates of the carcinoma have not significantly improved (30-32). Therefore, novel treatment need to be developed to add to the therapeutic strategies. Therefore, we developed and validated is a very attractive and promising modality for gene therapy.

In conclusion, multiple shRNA expression vector shows its advantages in silencing genes, compared with a single one. The results lay a solid foundation for multi-site RNAi therapy. This attractive strategy can be used to silence several genes by expression of different shRNAs targeting different genes and represents a powerful investigative and potentially therapeutic strategy in the prevention and treatment of cancer $(13,28,33-35)$. In addition, our results indicate that APRIL gene is related to the malignancy of carcinoma cells which can be reduced by APRIL knockdown. Thus, targeting APRIL using a multiple shRNA expression vector may serve as a novel therapeutic strategy for tumors.

\section{Acknowledgements}

This study was supported by grant XK200723 from Key Laboratory Subject of Jiangsu Province. We gratefully acknowledge the helpful participation of Feng Wang, Weifeng Ding, Rongrong Jing, Huoyan Ji and Fang Zhao.

\section{References}

1. Hahne M, Kataoka T, Schröter M, Hofmann K, Irmler M, Bodmer JL, Schneider P, Bornand T, Holler N, French LE, et al: APRIL, a new ligand of the tumor necrosis factor family, stimulates tumor cell growth. J Exp Med 188: 1185-1190, 1998.

2. Kimberley FC, van Bostelen L, Cameron K, Hardenberg G, Marquart JA, Hahne M and Medema JP: The proteoglycan (heparan sulfate proteoglycan) binding domain of APRIL serves as a platform for ligand multimerization and cross-linking. FASEB J 23: 1584-1595, 2009.

3. Deshayes F, Laprée G, Portier A, Richard Y, Pencalet P, MahieuCaputo D, Horellou P and Tsapis A: Abnormal production of the TNF-homologue APRIL increases the proliferation of human malignant glioblastoma cell lines via a specific receptor. Oncogene 23: 3005-3012, 2004

4. Sun B, Wang H, Wang X, Huang H, Ding W, Jing R, Shi G and Zhu L: A proliferation-inducing ligand: a new biomarker for non-small cell lung cancer. Exp Lung Res 35: 486-500, 2009.

5. Mhawech-Fauceglia P, Kaya G, Sauter G, McKee T, Donze O, Schwaller J and Huard B: The source of APRIL up-regulation in human solid tumor lesions. J Leukoc Biol 80: 697-704, 2006.

6. Planelles L, Medema JP, Hahne M and Hardenberg G: The expanding role of APRIL in cancer and immunity. Curr Mol Med 8: 829-844, 2008.

7. Wang F, Chen L, Mao ZB, Shao JG, Tan C and Huang WD: Lentivirus-mediated short hairpin RNA targeting the APRIL gene suppresses the growth of pancreatic cancer cells in vitro and in vivo. Oncol Rep 20: 135-139, 2008.

8. Moreaux J, Veyrune JL, De Vos J and Klein B: APRIL is overexpressed in cancer: link with tumor progression. BMC Cancer 9: 83, 2009.

9. Dillon SR, Gross JA, Ansell SM and Novak AJ: An APRIL to remember: novel TNF ligands as therapeutic targets. Nat Rev Drug Discov 5: 235-246, 2006.

10. Fire A,Xu S,Montgomery MK, Kostas SA,Driver SE and MelloCC: Potent and specific genetic interference by double-stranded RNA in Caenorhabditis elegans. Nature 391: 806-811, 1998.

11. Cogoni $\mathrm{C}$ and Macino G: Post-transcriptional gene silencing across kingdoms. Curr Opin Genet Dev 10: 638-643, 2000.

12. Sioud M: Therapeutic siRNAs. Trends Pharmacol Sci 25: 22-28, 2004.

13. Wang S, Shi Z, Liu W, Jules J and Feng X: Development and validation of vectors containing multiple siRNA expression cassettes for maximizing the efficiency of gene silencing. BMC Biotechnol 6: 50,2006

14. Ding W, Wang J, Sun B, Ju S, Yuan H, Wang X, Wang Y and Wang H: APRIL knockdown suppresses migration and invasion of human colon carcinoma cells. Clin Biochem 42: 1694-1698, 2009.

15. Hendriks J, Planelles L, de Jong-Odding J, Hardenberg G, Pals ST, Hahne M, Spaargaren M and Medema JP: Heparan sulfate proteoglycan binding promotes APRIL-induced tumor cell proliferation. Cell Death Differ 12: 637-648, 2005.

16. Rennert P, Schneider P, Cachero TG, Thompson J, Trabach L, Hertig S, Holler N, Qian F, Mullen C, Strauch K, Browning JL, Ambrose $\mathrm{C}$ and Tschopp J: A soluble form of $\mathrm{B}$ cell maturation antigen, a receptor for the tumor necrosis factor family member APRIL, inhibits tumor cell growth. J Exp Med 192: 1677-1684, 2000.

17. Marsters SA, Yan M, Pitti RM, Haas PE, Dixit VM and Ashkenazi A: Interaction of the TNF homologues BLyS and APRIL with the TNF receptor homologues BCMA and TACI. Curr Biol 10: 785-788, 2000.

18. Wu Y, Bressette D, Carrell JA, Kaufman T, Feng P, Taylor K, Gan Y, Cho YH, Garcia AD, Gollatz E, et al: Tumor necrosis factor (TNF) receptor superfamily member TACI is a high affinity receptor for TNF family members APRIL and BLyS. J Biol Chem 275: 35478-35485, 2000.

19. Esko JD and Lindahl U: Molecular diversity of heparan sulfate. J Clin Invest 108: 169-173, 2001.

20. Gallagher JT: Heparan sulfate: growth control with a restricted sequence menu. J Clin Invest 108: 357-361, 2001.

21. Perrimon $\mathrm{N}$ and Bernfield M: Specificities of heparan sulphate proteoglycans in developmental processes. Nature 404: 725-728, 2000 . 
22. Hayashida K, Johnston DR, Goldberger O and Park PW: Syndecan-1 expression in epithelial cells is induced by transforming growth factor beta through a PKA-dependent pathway. J Biol Chem 281: 24365-24374, 2006.

23. Ding W, Ju S, Jiang S, Zhu L, Wang Y and Wang H: Reduced APRIL expression induces cellular senescence via a HSPGdependent pathway. Pathol Oncol Res 15: 693-701, 2009.

24. Hannon GJ: RNA interference. Nature 418: 244-251, 2002.

25. Dykxhoorn DM: RNA interference as an anticancer therapy: a patent perspective. Expert Opin Ther Pat 19: 475-491, 2009.

26. Wang SL, Yao HH and Qin ZH: Strategies for short hairpin RNA delivery in cancer gene therapy. Expert Opin Biol Ther 9: 1357-1368, 2009.

27. Gou D, Weng T, Wang Y, Wang Z, Zhang H, Gao L, Chen Z, Wang P and Liu L: A novel approach for the construction of multiple shRNA expression vectors. J Gene Med 9: 751-763, 2007.

28. Yan Y, Zhang J, Guo JL, Huang W and Yang YZ: Multiple shRNA-mediated knockdown of TACE reduces the malignancy of HeLa cells. Cell Biol Int 33: 158-164, 2009.

29. Jemal A, Siegel R, Xu J and Ward E: Cancer statistics, 2010. CA Cancer J Clin 60: 277-300, 2010.
30. Center MM, Jemal A, Smith RA and Ward E: Worldwide variations in colorectal cancer. CA Cancer J Clin 59: 366-378, 2009.

31. Ortega J, Vigil CE and Chodkiewicz C: Current progress in targeted therapy for colorectal cancer. Cancer Control 17: 7-15, 2010.

32. Markowitz SD and Bertagnolli MM: Molecular origins of cancer: Molecular basis of colorectal cancer. N Engl J Med 361: 2449-2460, 2009.

33. Jazag A, Kanai F, Ijichi H, Tateishi K, Ikenoue T, Tanaka Y, Ohta M, Imamura J, Guleng B, Asaoka Y, et al: Single smallinterfering RNA expression vector for silencing multiple transforming growth factor-beta pathway components. Nucleic Acids Res 33: e131, 2005.

34. ter Brake O, Konstantinova P, Ceylan M and Berkhout B: Silencing of HIV-1 with RNA interference: a multiple shRNA approach. Mol Ther 14: 883-892, 2006.

35. Song J, Giang A, Lu Y, Pang S and Chiu R: Multiple shRNA expressing vector enhances efficiency of gene silencing. BMB Rep 41: 358-362, 2008. 